\title{
Low Energy X-Ray Detection with a Silicon Multi-Cathode Detector for Microanalysis
}

\author{
S. Barkan*, V. D. Saveliev*, C. R. Tull*, L. Feng*, M. Takahashi*, N. Matsumori*, \\ D. E. Newbury**, J. A. Small** and J. S. Iwanczyk*** \\ *SII NanoTechnology USA Inc., 19355 Business Dr., Suite 9, Northridge, CA 91324, USA \\ **National Institute of Standards and Technology, 100 Bureau Dr., Gaithersburg, MD 20899, USA \\ ***Photon Imaging, Inc., 19355 Business Center Dr., Suite 10, Northridge, CA 91324, USA
}

This paper presents several aspects of our design efforts toward the development of a large-area, high energy resolution analytical x-ray spectrometry system for x-ray microanalysis and x-ray spectrum imaging [1]. The spectrometer achieves excellent energy resolution and is capable of operating at very high counting rates (up to $600,000 \mathrm{cps}$ throughput). The Vortex-EM ${ }^{\mathrm{TM}}$ spectrometer is based on a thermoelectrically-cooled silicon multi-cathode detector (SMCD), which is a type of "drift" detector [2-3]. The detector is specifically designed for optimal performance in the $0.2-40 \mathrm{keV}$ range. Recent advancements in the detector design enhance the low energy $\mathrm{x}$-ray performance. The spectrometer utilizes a non-cryogenic design, operating with thermoelectric cooling and passive heat transfer to the ambient without using any moving parts; a photograph of the spectrometer is shown in Figure 1.

With the current detector, which is $\sim 50 \mathrm{~mm}^{2}$ active area and $\sim 0.35 \mathrm{~mm}$ thick, an energy resolution of $125 \mathrm{eV}$ FWHM is achievable (at $5.9 \mathrm{keV}, 6 \mu \mathrm{s}$ amplifier peaking time), with a peak-tobackground ratio of 2000:1 in an ${ }^{55} \mathrm{Fe}$ spectrum. These performance characteristics are superior to any non-cryogenic solid state detector of similar dimensions. The throughput rate is $\sim 600 \mathrm{kcps}$ for an input rate of $1.5 \mathrm{Mcps}$, at $0.25 \mu$ s peaking time. The detector shows virtually no change in energy resolution, nor shift in peak position as a function of count rate.

The Vortex-EM ${ }^{\mathrm{TM}}$ spectrometer was integrated into a JEOL 840 SEM at the National Institutes of Standard and Technology, and the spectral performance was evaluated in response to a variety of samples. Examples of spectra from boron, calcium carbonate, silicon dioxide and silicon nitride samples are shown in Figures $2-5$, respectively. Additional low energy x-ray spectra and low energy performance parameters in electron microscopy applications will be presented.

[1] 1. S. Barkan, V. Saveliev, J. Iwanczyk, L. Feng, C. Tull, B. Patt, D. Newbury, J. Small, N. Zaluzec, "A New Improved Silicon Multi-Cathode Detector (SMCD) for Microanalysis and X-Ray Mapping Applications”, Microscopy Today, November 2004.

[2] J. S. Iwanczyk, B.E. Patt, C.R. Tull, J.D. Segal, C.J. Kenney, J. Bradley, B. Hedman, and K.O. Hodgson, "Large Area silicon Drift Detectors for X-Rays- New Results", IEEE Trans. Nucl. Sci., 46 (1999) 284-288.

[3] S. Barkan, J.S. Iwanczyk, B.E. Patt, L. Feng, C.R. Tull and G. Vilkelis, "Vortex ${ }^{\text {TM }}$ - A new high performance silicon drift detector for XRD and XRF Applications", Advances in X-Ray Analysis, 46 (2003) 332-337. 


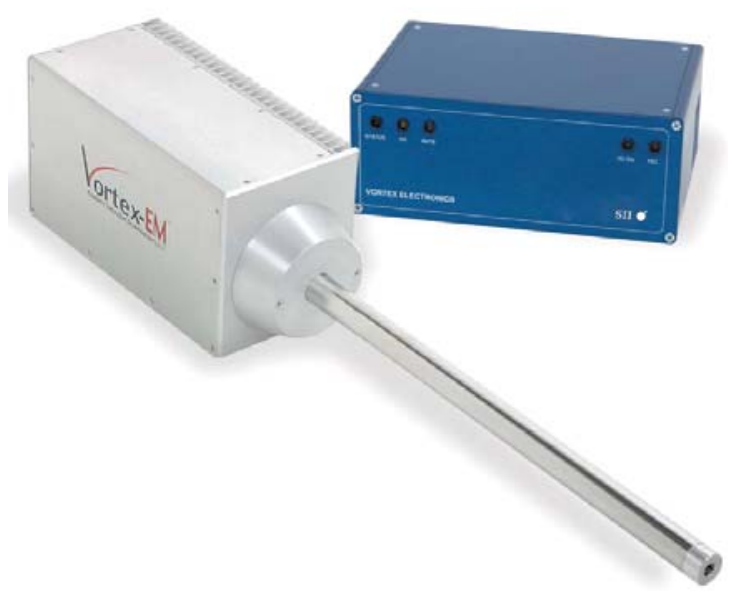

Fig. 1. Vortex-EM ${ }^{\mathrm{TM}}$ spectrometer and digital pulse processor; snout length is $300 \mathrm{~mm}$.

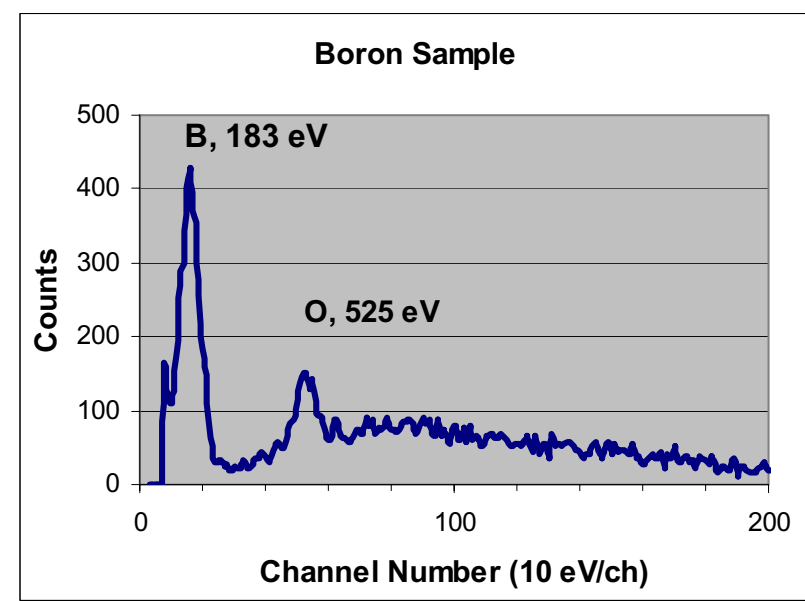

Fig. 2. Spectrum from a boron sample in the electron microscope.

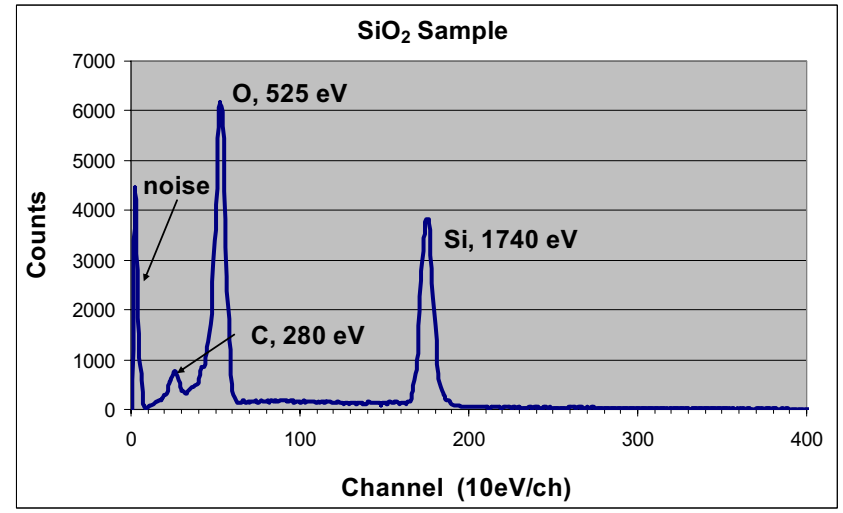

Fig. 4. Spectrum from a $\mathrm{SiO}_{2}$ sample in the electron microscope.

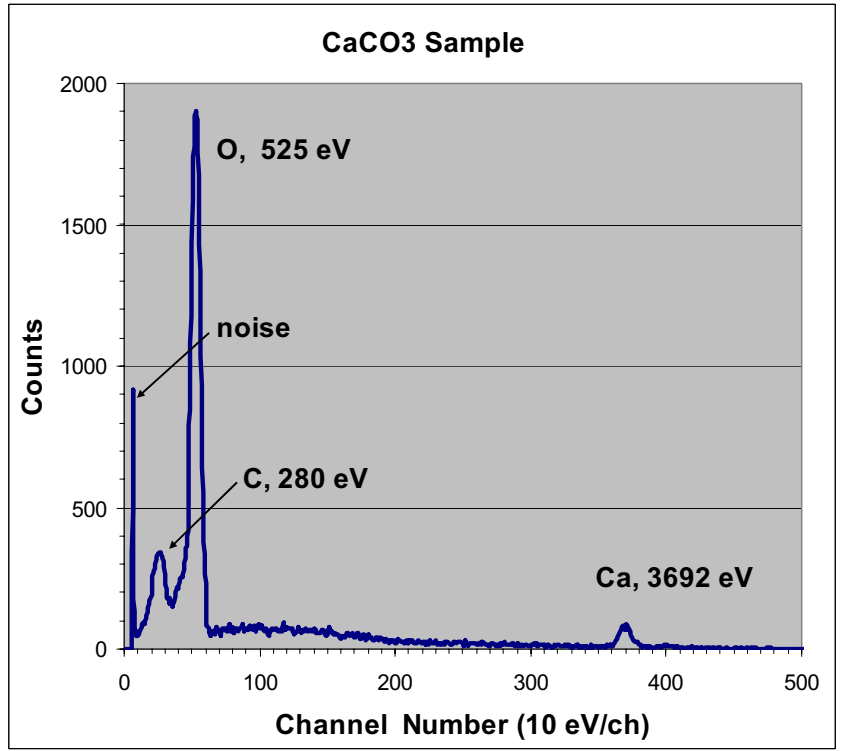

Fig. 3. Spectrum from a $\mathrm{CaCO}_{3}$ sample in the electron microscope.

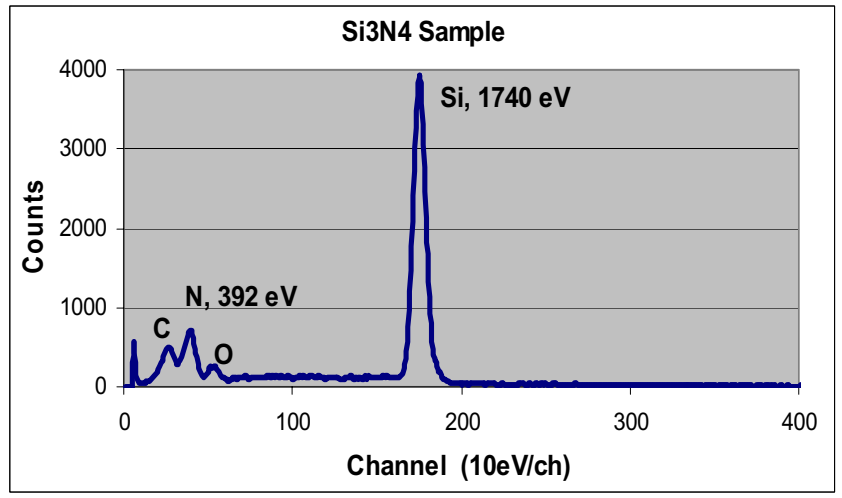

Fig. 5. Spectrum from a $\mathrm{Si}_{3} \mathrm{~N}_{4}$ sample in the electron microscope. 